\title{
Os benefícios do aleitamento materno precoce
}

\author{
The benefits of early breastfeeding \\ Los beneficios de la lactancia materna temprana
}

Recebido: 17/09/2021 | Revisado: 21/09/2021 | Aceito: 24/09/2021 | Publicado: 25/09/2021

\author{
Ana Beatriz Pereira de Almeida \\ ORCID: https://orcid.org/0000-0002-3469-953X \\ Centro Universitário Fametro, Brasil \\ E-mail: ana_beatrizalmeida@hotmail.com \\ Wayne Thayná Ozório \\ ORCID: https://orcid.org/0000-0002-1879-2320 \\ Centro Universitário Fametro, Brasil \\ E-mail: ozoriowayne@gmail.com \\ José Carlos de Sales Ferreira \\ ORCID: https://orcid.org/0000-0002-1867-8229 \\ Centro Universitário Fametro, Brasil \\ E-mail: jcarlos.sales@gmail.com
}

\begin{abstract}
Resumo
Introdução: o leite materno é de suma importância para o desenvolvimento do bebê e proteção contra doenças alérgicas, desnutrição, diabetes melittus, doenças digestivas, obesidade, cáries, entre outras, pois sua composição contém proteína, lipídio, carboidrato. Objetivo Geral: analisar a importância do aleitamento materno no desenvolvimento do bebê, os benefícios para saúde da mãe e os motivos do desmame precoce. Metodologia: Para a realização desse estudo optou-se por uma pesquisa na modalidade de revisão integrativa de literatura. A revisão integrativa determina o conhecimento atual sobre uma temática específica, já que é conduzida de modo a identificar, analisar e sintetizar resultados de estudos independentes sobre o mesmo assunto. Resultados e Discussão: A amamentação corresponde a uma das etapas mais importantes no processo reprodutivo da mulher e sua prática oferece benefícios tanto para mãe como para o recém-nascido. Considerações Finais: o aleitamento materno é um alimento de suma importância para a sobrevivência do lactante, pois é o único que oferece nutrientes adequados, em que em nenhum outro leite industrializado pode ser encontrado.
\end{abstract}

Palavras-chave: Amamentação; Leite humano; Leite materno; Nutrientes.

\begin{abstract}
Introduction: breast milk is extremely important for the baby's development and protection against allergic diseases, malnutrition, diabetes mellitus, digestive diseases, obesity, caries, among others, because its composition contains protein, lipid, carbohydrate. General Objective: to analyze the importance of breastfeeding in the baby's development, the benefits for the mother's health and the reasons for early weaning. Methodology: To carry out this study, we opted for a research in the modality of integrative literature review. The integrative review determines the current knowledge on a specific topic, as it is conducted in order to identify, analyze and synthesize results of independent studies on the same subject. Results and Discussion: Breastfeeding is one of the most important steps in a woman's reproductive process and its practice offers benefits for both the mother and the newborn. Final Considerations: Breastfeeding is a food of paramount importance for the survival of the infant, as it is the only one that offers adequate nutrients, in which no other industrialized milk can be found.
\end{abstract}

Keywords: Breastfeeding; Human milk; Breast milk; Nutrients.

\section{Resumen}

Introducción: la leche materna es de suma importancia para el desarrollo y protección del bebé frente a enfermedades alérgicas, desnutrición, diabetes mellitus, enfermedades digestivas, obesidad, caries, entre otras, debido a que su composición contiene proteínas, lípidos, carbohidratos. Objetivo general: analizar la importancia de la lactancia materna en el desarrollo del bebé, los beneficios para la salud de la madre y los motivos del destete precoz. Metodología: Para la realización de este estudio se optó por una investigación en la modalidad de revisión integradora de la literatura. La revisión integradora determina el conocimiento actual sobre un tema específico, ya que se realiza con el fin de identificar, analizar y sintetizar resultados de estudios independientes sobre el mismo tema. Resultados y discusión: La lactancia materna es uno de los pasos más importantes en el proceso reproductivo de una mujer y su práctica ofrece beneficios tanto para la madre como para el recién nacido. Consideraciones finales: La lactancia materna es un alimento de suma importancia para la supervivencia del lactante, ya que es el único que ofrece los nutrientes adecuados, en el que no se puede encontrar ninguna otra leche industrializada.

Palabras clave: Amamantamiento; Leche humana; La leche materna; Nutrientes. 


\section{Introdução}

A amamentação é um ato sublime que cria um gesto afetivo entre a mãe e o bebê, além disso, promove vários benefícios ao desenvolvimento do bebê. Sua composição de nutrientes é completa sendo considerado o alimento ideal, trazendo os benefícios do desenvolvimento facial, emocional e imunológico (Brasil, 2015).

De acordo com Leite et al., (2016), o contato precoce entre mãe e bebê deve ser valorizado pois alcança vários objetivos, dentre eles a capacidade para amar do ser humano que se dá logo após o nascimento, trazendo benefício curto e longo prazo.

No aspecto psicossocial, o processo de amamentação está relacionado ao binômio mãe e filho. De acordo com a Organização Mundial da Saúde (OMS) (2005), a prática do aleitamento deve ser exclusiva até os seis meses de vida do bebê.

O aleitamento Materno exclusivo é indicado ser iniciado na sala do parto logo nas primeiras horas, e é também recomendo até os seis meses de vida, é ressaltado por Andrade (2014) que é pacifico o fato de que o aleitamento materno deva ser iniciado após o parto uma vez que o colostro e considerado a primeira imunização do neonato pela presença de imunoglobulinas e maior quantidade de proteínas e vitamina A.

Sendo assim, é importante enfatizar que o leite materno é de suma importância para o desenvolvimento do bebê e proteção contra doenças alérgicas, desnutrição, diabetes melittus, doenças digestivas, obesidade, cáries, entre outras, pois sua composição contém proteína, lipídio, carboidrato (Costa et al., 2013).

Em concordância a isso, Leite et al., (2016) relata que o leite materno por oferecer uma proteção imunológica e ser considerado uma nutrição ideal e essencial é que se deve por parte do profissional de saúde, incentivar a amamentação. Já em relação aos benefícios da mãe na amamentação é importante para auxiliar na diminuição do sangramento logo que o bebê nasce e atuante na prevenção do câncer de mama e ovário (Costa et al., 2013).

O profissional de saúde tem um papel fundamental na promoção do aleitamento materno exclusivo e deve ser um instrumento para que as mulheres possam adquirir autonomia no agir. Compreende-se que ao se estabelecer um contato imediato entre mãe e filho ainda em sala de parto faz com que a equipe de enfermagem pratique um papel importante como autora e mediadora das ações e passos da IHAC (Leite et al., 2016; Marciel et al., 2013).

O leite materno por oferecer uma proteção imunológica e ser considerado uma nutrição ideal e essencial é que se deve, por parte do profissional de saúde, incentivar a amamentação, desta forma, o desenvolvimento deste estudo justifica-se pela necessidade de se compreender acerca da temática abordada, tendo em vista sua importância incontestável tanto para o desenvolvimento materno-infantil.

Desta forma, o objetivo geral desse estudo é analisar a importância do aleitamento materno no desenvolvimento do bebê, os benefícios para saúde da mãe e os motivos do desmame precoce.

\section{Metodologia}

Para a realização desse estudo optou-se por uma pesquisa na modalidade de revisão integrativa de literatura. A revisão integrativa determina o conhecimento atual sobre uma temática específica, já que é conduzida de modo a identificar, analisar e sintetizar resultados de estudos independentes sobre o mesmo assunto (Silveira et al., 2008).

A Revisão Integrativa é um método de pesquisa apontado como ferramenta de grande relevância no campo da saúde, por proporcionar a busca, a avaliação crítica e a síntese de evidências sobre um tema investigado. Esses aspectos facilitam a identificação dos resultados relevantes, de lacunas que direcionam para o desenvolvimento de futuras pesquisas e auxiliam o profissional a escolher condutas e a tomar decisões, proporcionando um saber crítico (Souza, Silva \& Carvalho, 2010). 
Para extrair os dados dos artigos selecionados, será necessária a utilização de um instrumento previamente elaborado capaz de assegurar que a totalidade dos dados relevantes seja extraída, minimizar o risco de erros na transcrição, garantir precisão na checagem das informações e servir como registro (Souza, Silva \& Carvalho, 2010).

A coleta das informações para a pesquisa bibliográfica será por meio da exploração da base de dados da Biblioteca Virtual em Saúde (BVS), Biblioteca Cientifica Eletrônica Virtual (SCIELO) e Literatura Latino - Americana e do Caribe em Ciências da Saúde (LILACS).

A busca na base de dados será orientada pelos descritores: Vitamina D, doenças autoimunes, ação fisiológica; e será realizado em todos os índices, buscando captar o maior número de artigos publicados no período proposto que abordem a temática em discussão, visando o desenvolvimento fidedigno desse estudo.

Para uma análise crítica e reflexiva dos estudos incluídos na revisão, será realizada uma leitura minuciosa e criteriosa destacando os que atingirem os critérios de inclusão e que contemplarem o objetivo proposto, para viabilizar o resultado da pesquisa de forma objetiva.

Serão incluídos os artigos publicados em língua portuguesa nos últimos dez anos, com texto completo, disponível online, com acesso livre. Serão excluídos da amostra os artigos publicados em línguas estrangeiras, os que não apresentarem o texto na integra, artigos que não apresentavam relação direta com o tema, resumos, monografias, dissertações, teses e artigos repetidos.

\section{Resultados e Discussão}

\subsection{Descrever a Importância da Amamentação}

$\mathrm{O}$ aleitamento materno exclusivo reduz o risco de asma, e esse efeito protetor irá permanecer pelo menos durante a primeira década de vida da criança, sendo particularmente evidente em crianças com história familiar de doenças a tópicas. Do ponto de vista epigenético, tem no leite materno a fonte ideal de nutrição, permitindo que todo o seu potencial genético inerente seja atingido. Isso ocorre porque sua composição garante as quantidades necessárias de água, carboidratos, lipídeos e proteínas para o desenvolvimento adequado da criança (Nunes, 2015; Gnoatto et al., 2018).

A amamentação tem ação importante para o lactente na proteção contra infecções, diarreia, doenças respiratórias, autoimunes, celíaca e de Crohn, linfomas, diabetes mellitus, entre outras. No primeiro ano de vida da criança, a maneira mais eficiente é a amamentação, dando desenvolvimento e atendendo a todos os aspectos psicológicos, nutricionais e imunológicos. O aleitamento materno é a mais importante e eficaz estratégia para a saúde da criança, reforçando vínculo afeto e proteção, além de garantir a nutrição adequada (Mendes et al., 2019; Barbieri et al., 2015; Silva et al., 2020).

O leite materno (LM) é o alimento mais completo, pois contém vitaminas, sais minerais e nutrientes necessários ao crescimento e desenvolvimento da criança, pois favorece a construção de vínculo afetivo, e também traz benefícios a mãe evita complicações hemorrágicas e entre outros. Com menor risco de contaminação, a proteção imunológica e o valor nutricional contribuem para a redução de mortalidade infantil, por infecção respiratória e por diarreia (Silva et al., 2020; Tessari et al., 2019).

Na visão das puérperas, em especial as primíparas, as orientações ofertadas por profissionais de saúde têm um impacto considerável sobre o aleitamento materno, podendo determinar inclusive a continuidade do tempo de amamentação e a superação dos desafios sentidos no processo do amamentar (Santana et al., 2019).

A amamentação corresponde a uma das etapas mais importantes no processo reprodutivo da mulher e sua prática oferece benefícios tanto para mãe como para o recém-nascido. Marciel et al., (2013), ressalta que amamentação proporciona ao recém-nascido crescimento e desenvolvimento saudáveis, e também fortalece o vínculo entre mãe e filho. Em relação aos 
benefícios do aleitamento materno para as mães, sabe-se que a prática parece reduzir alguns tipos de fraturas ósseas, câncer de mama e de ovários, além de diminuir o risco de morte por artrite reumatoide.

O aleitamento materno é um processo fisiológico que traz inúmeras vantagens biopsicosocial para as puérperas. Permite ainda um grandioso impacto na promoção da saúde integral da mãe e do bebê, repercutindo nos indicadores de saúde de toda a sociedade (Mendes et al, 2019).

Protegendo o aleitamento materno exclusivo, reduz-se a morbimortalidade materna e infantil, aumenta-se o quociente de inteligência (QI) infantil e o desempenho escolar, contribuindo assim indiretamente para a redução da pobreza (Rimes et al., 2019).

O AM contribui com o meio ambiente, pois, ao contrário dos leites artificiais, não agride e nem polui, além de já ser naturalmente preparado na temperatura ideal para o bebê com todos os nutrientes necessários, sem gasto de energia, de maneira eficaz. Já o leite artificial prejudica no crescimento e desenvolvimento, podendo levá-la tanto à desnutrição quanto à obesidade (Andrade et al., 2018).

\subsection{Malefícios do desmame precoce}

Alergia alimentar tem sido encontrada menos frequentemente em crianças amamentadas exclusivamente no peito, e a dermatite atópica pode ter o seu início retardado com a alimentação natural, algumas infecções geralmente são evitadas com o aleitamento materno como: diarreia, pneumonia, bronquites, gripe, paralisia infantil, infecções urinárias, otite e infecção no trato intestinal (Silva, 2020).

O desmame precoce resulta em alterações negativas na saúde e desenvolvimento dos bebês, como maiores propensões a diarreias, alergias alimentares e desenvolvimento motor-oral incompleto da criança. Algumas patologias como a desnutrição, obesidade infantil, riscos ao trato digestivo e vias respiratórias podem ser advindas com a introdução precoce de alimentos (Nunes et al., 2020).

A principal causa para o desenvolvimento da alergia a proteína do leite de vaca (APLV) é a introdução precoce do leite de vaca na alimentação infantil, principalmente lactentes (Sampaio et al., 2017). Silva (2020) ressalta que prática do desmame precoce resulta também em maiores taxas de propensão a alergias alimentares, as mães tendem a acreditar que o leite de vaca é melhor que o seu próprio leite, e acabam introduzindo-o muito cedo.

De acordo com as alergias respiratórias, a incidência de crianças que não foram amamentadas exclusivamente até o $6^{\circ}$ mês foi maior do que as que foram amamentados exclusivamente, isto ocorreu em atributo das eminentes quantidades de anticorpos (Nunes et al., 2020).

A imaturidade do sistema imunológico dos neonatos está diretamente relacionada à sensibilização alérgica, correlacionando o aparecimento de alergias em crianças que foram privadas do aleitamento materno exclusivo, surgindo outras consequências à saúde (José et al., 2017).

\subsection{O leite materno como aporte nutricional}

O Aleitamento Materno Exclusivo (AME) consiste no mais nutritivo e adequado alimento para a criança até os seis primeiros meses de vida, por ser rico em vitaminas, proteínas, carboidratos, gorduras, sais minerais e água. Ele é composto por 160 substâncias, representado por proteínas, gorduras, carboidratos e células (Silva et al., 2017).

As proteínas do leite humano incluem caseína e lactalbuminas. A alfalactoalbumina é encontrada em maior concentração e apresenta potencial alergênico praticamente nulo, outra proteína encontrada no leite humano é a leptina, um hormônio que possui a ação de inibir o apetite e as vias anabólicas e estimular as vias catabólicas, contribuindo assim para a prevenção da obesidade em crianças que recebem aleitamento materno exclusivo (Filho et al., 2020). 
A fração lipídica deste alimento representa a maior fonte de energia para crianças em aleitamento, contribuindo com $40 \%$ a $55 \%$ do total de energia ingerida e provê nutrientes essenciais, como as vitaminas lipossolúveis (vitaminas A, D, E e K) (Clemente et al., 2016).

Até o $7^{\circ}$ dia após o parto, o LH é considerado colostro, entre o $8^{\circ}$ e $14^{\circ}$ dia pós-parto é considerado leite de transição e após o $15^{\circ}$ dia pós-parto passa a ser considerado leite maduro (Rydlewsi et al., 2020).

O colostro possui um fator de crescimento que promove a colonização do trato gastrointestinal infantil pelas bifidobactérias ou lactobacilos, que estimulam a produção de ácido lático, prejudicial ao desenvolvimento de organismos exógenos patogênicos, envolvidos na gênese de infecções. O colostro é um líquido amarelado e viscoso, possui alta densidade, contém menos gordura, vitaminas hidrossolúveis, lactose e mais proteínas, vitaminas lipossolúveis, e minerais como zinco e sódio, e o colostro evolui para o leite maduro (Borges et al., 2020).

Santos (2018), ressalta que os minerais constituintes do leite materno maduro são altamente disponíveis e suas concentrações não são afetadas pela alimentação da mãe, os quais são: Cálcio, Ferro, Zinco e Cobre. Os minerais podem ser encontrados na forma de sódio, potássio, cloreto, cálcio, magnésio, fósforo ferro, flúor, zinco, cobre, manganês, selênio e iodeto.

\section{Conclusão}

O aleitamento materno é um elemento de fundamental importância para a saúde do bebê, é através de dele que acontece um aumento do vínculo materno-infantil, diminuindo os índices de óbito e enfermidades em grande maioria. No leite humano há nutrientes essenciais para o desenvolvimento do recém-nascido e fatores protetores de doenças, sendo indispensável.

É de responsabilidade dos profissionais da saúde orientar, estimular, incentivar e conscientizar as mães sobre os benefícios do aleitamento materno. Além disso, esses devem ser multiplicadores do aleitamento, repassando informações e orientando a melhor maneira de amamentar, de forma que esse processo ocorra da forma mais natural e sem intercorrências para a mãe e o bebê.

Diante do exposto o aleitamento materno é um alimento de suma importância para a sobrevivência do lactante, pois é o único que oferece nutrientes adequados, em que em nenhum outro leite industrializado pode ser encontrado. Porém a amamentação materna infantil oferece vantagens para a saúde da mãe trazendo o conforto e o bem-estar para o lactente.

\section{Referências}

Acioli, S. A (2018). Prática educativa como expressão do cuidado em saúde pública. Rev. bras. enferm. 61(1), 117-21.

Andrade, I. S. N. (2014). Aleitamento materno e seus benefícios: primeiro passo para a promoção saúde. Revista Brasileira Promoção Saúde, Fortaleza, 27(2), $149-150$.

Andrade, H., Pessoa, R., \& LCV, D. (2018). Fatores relacionados ao desmame precoce do aleitamento materno. Revista Brasileira Medicina de Família e Comunidade, 1-11.

Barbieri, M., Bercini, L., Brondani, K., Ferrari, R., Tacla, M., \& Sant'anna, F. (2015). Aleitamento materno: orientações recebidas no pré-natal, parto e puerpério. Semina: Ciências Biológicas e da Saúde, Londrina, 36(1), 17-24.

Barbosa, J., Santos, F., \& Silva, P. (2013). Fatores associados à baixa adesão ao aleitamento materno exclusivo e ao desmame precoce. Revista Tecer - Belo Horizonte - 6(11).

Boccolini, C. C., \& Oliveira, M. (2015). Fatores associados ao aleitamento exclusivo nos primeiros seis meses de vida no Brasil: revisão sistemática. Revista Saúde Pública.

Borges, C., Duarte, C., Gemelli, C., Santana, J., \& Mendes, R. (2020). Valor calórico do leite humano ordenhado pasteurizado de um banco de leite. Brazilian Journal of Development, Curitiba, 6(3), 14243-14258. 
Brasil., \& Ministério Da Saude. Secretaria De Atenção Á Saúde, D. D. (2015). Aleitamento materno e alimentação complementa. Cadernos de Atenção Básica, 23, Departamento de Atenção Básica. - 2. ed. - Brasília, 1-184.

Campos, D., Araújo, N., Silva, T., Machado, A., \& Soares, L. (2018). Aleitamento materno na prevenção contra infecções gastroentéricas . Saber Científico, Porto Velho, 7(2), 68-75.

Carvalho, M. R., \& Tamez, R. (2016). Amamentação: bases científicas para pratica profissional. Rio de Janeiro: Guanabara Koogan.

Capucho. L. B., Forechi L., Lima, R. C. D., et al. (2017). Fatores que interferem na amamentação exclusiva. Rev. Bras. Pesq. Saúde, Vitória, 19(1), 108-113.

Clemente, H., Melo, L., Ramalho, H., Rodrigues, K., \& Dimenstein, R. (2016). Vitamina E no soro e colostro materno e o requerimento nutricional do lactente. RBM Revista Brasileira de Medicina, 75-80.

Costa, A., \& C.M, S. (2010). Modulação e composição de ácidos graxos do leite humano. Revista de Nutrição Campinas, $23(3)$, $445-457$.

Costa, L., Queiroz, L., Queiroz, R., Ribeiro, T., \& Fonseca, M. (2013). Importância do aleitamento materno exclusivo: uma revisão sistemática da literatura. Revista Ciênc. Saúde São Luís, 15(1), 39-46.

Cunha, É. S. (2016). Aleitamento Materno: Contribuições da Enfermagem. Ensaios e Ciência: Ciências Biológicas, Agrárias e da Saúde, 20(2), 86-92.

Diogo, E., Souza, T., \& Zocche, D. (2011). Causas do desmame precoce e suas interfaces com a condição socioeconômica e escolaridade. Enfermagem em Foco, 10-13.

Duncan, B.B., Schmidt M. I., \& Giugliani, E. R. J. (2014). Medicina ambulatorial: condutas de atenção primária baseadas em evidências. $3^{\text {a }}$ ed. Porto Alegre: Artes Médicas.

Esteves, T., Daumas, R., Oliveira, M., Andrade, C., \& Leite, I. (2014). Fatores associados à amamentação na primeira hora de vida: revisão sistemática. Revista Saúde Pública; 48(4), 697-703.

Escobar, M. Y., Teruya, P. Y., Grisi S., et al. (2016). Aleitamento materno e condições socioeconômico-culturais: fatores que levamao desmame precoce. Rev Bras Saúde Matern Infant. 2(3), 253-61.

Fujimore E., \& Ohara, C. V. S. (2019). Enfermagem e a saúde da criança na atenção básica. São Paulo: Manole.

Favoretto, M., Vieczorek, A., Silva, C., Peder, L., \& Teixeira, J. (2016). Composição Lipídica E Proteica Do Leite Humano Pré E Pós-Pasteurização. Visão Acadêmica, Curitiba, 17(4), 43-55.

Ferreira, H., Oliveira, M., Bernado, E., Almeida, P., Aquino, P., \& Pinheiro, A. (2018). Fatores Associados à Adesão ao Aleitamento Materno Exclusivo. Ciência \& Saúde Coletiva, 23(3), 683-690.

Ferreira, M., Nelas, P., \& Duarte, J. (2011). Motivação para o Aleitamento Materno: Variáveis Intervenientes. Millenium, 40, $23-38$.

Filho, L., Silva, A., Pereira, C., Ferreira, D., Diniz, I., Quinto, M., \& Belo, V. (2020). A amamentação como prevenção da obesidade infantil: Uma revisão narrativa. Brazilian Journal of health Review , Curitiba, 3(4), 11146-11162.

Freitas, L., Barreto, C., Soare, C., \& Carvalho, B. (2020). Percepção Dos Enfermeiros Acerca Da Composição E Características Do Leite Humano. Journal of Medicine and Health Promotion, 157-168.

Freitas, R., Macedo, M., Lessa, A., Ferraz, V., Soares, N., Martins, B., \& Teixeira, R. (2019). Composição em ácidos graxos do leite maduro de nutrizes. Revista Bras. Saúde Mater. Infant., Recife, 19 (4), 827-836.

Gomes, R., Silva, P., Silva, C., Santos, J., Macário, F., Ramos, F., \& Sousa, J. (2020). Fatores que levam ao desmame precoce do aleitamento materno. Brazilian Journal of Development, Curitiba, 6(12), 100688-100700.

José, D., Vitiato, J., Hass, K., França, T., \& Cavagnari, M. (2017). Relação Entre Desmame Precoce E Alergias Alimentares. Visão Acadêmica, Curitiba, 17(3).

Junior, A. R. F., Rocha, F. A. A., Souza, M. T. A., et al. (2016). Cuidado de enfermagem sobre a importância do Aleitamento Materno exclusivo: percepção de puérperas. Tempus, actas de saúde colet, Brasília, 10(3), 19-29.

Lamounier, J.A. (2018). Promoção e incentivo ao aleitamento materno: iniciativa hospital Amigo da Criança. J. Pediatr., Rio de Janeiro, 72, $365-368$.

Leite, M. F., Barbosa, P. A., Olivindo, D. D., \& Ximenes, V. D. (2016). Promoção do aleitamento materno na primeira hora de vida do recém-nascido por profissionais da enfermagem. Arq. Cienc. Saúde UNIPAR, Umuarama, 20(2), 137-143.

Maciel, A., Gondim, A., Silva, A., Barros, F., Barbosa, G., \& K.C., A. (2013). Conhecimento de gestantes e lactantes sobre aleitamento materno exclusivo. Revista Brasileira em Promoção da Saúde, 26(3), 311-317.

Margotti, E., \& Matielo, R. (2016). Fatores de risco para o desmame precoce. Revista da Rede de Enfermagem do Nordeste, 17(4), 537-544.

Marques, E., Cotta, R., \& Priore, S. (2011). Mitos e crenças sobre o aleitamento materno. Ciência \& Saúde Coletiva, 16(5), 2461-2468.

Martins, M., \& L. S, S. (2013). Benefícios da amamentação para saúde materna. Interfaces Científicas -Saúde e Ambiente, Aracaju, 1(3), 87-97.

Mendes, S., Lobo, I., Sousa, S., \& Vianna, R. (2019). Fatores relacionados com uma menor duração total do aleitamento materno. Ciência \& Saúde Coletiva, 24(5), 1821-1829. 
Research, Society and Development, v. 10, n. 12, e427101220741, 2021 (CC BY 4.0) | ISSN 2525-3409 | DOI: http://dx.doi.org/10.33448/rsd-v10i12.20741

Modes, P., Gaiva, M., Braun, A., \& Santos, V. (2020). Oficina educativa sobre aleitamento materno para gestantes de uma unidade saúde da família. Revista Eletrônica Acervo Saúde / Electronic Journal Collection Health , 1-10.

Monteiro, J., A.M.S., N., \& F.A, G. (2011). O aleitamento materno enquanto uma prática construída. Reflexões acerca da evolução histórica da amamentação e desmame precoce no Brasil. Invest Educ Enferm. 29(2), 315 - 321.

Nascimento, A., Silva, P., Nascimento, M., Souza, G., Calsavara, R., \& Santos, A. (2019). Atuação do enfermeiro da estratégia saúde da família no incentivo ao aleitamento materno durante o período pré-natal. Revista Eletrônica Acervo Saúde / Electronic Journal Collection Health. 21, 1-8.

Nunes, L. M. (2015). Importância do aleitamento materno na atualidade. Boletim Científico de Pediatria, 4(3), 55-8.

Nunes, L., \& Riquette, R. (2020). Associação entre o estado nutricional e alergias presentes em crianças que tiveram diferentes tipos de amamentação nos primeiros seis meses de vida. Revista Projeção Saúde e Vida. 1(2), 27.

Oliveira, M., \& Fanaro, G. (2015). Aleitamento materno na prevenção de sobrepeso, obesidade infantil e alergias. Revista Brasasileira Nutriçao Clinica, 32837 .

Passanha, A., Cervato-Mancuso, A., \& Memp, S. (2010). Elementos protetores do leite materno na prevenção de doenças gastrintestinais e respiratórias. Revista Bras Crescimento Desenvolvimento Humano, 351-360.

Rimes, K., Oliveira, M., \& Bocolli, C. (2019). Licença-maternidade e amamentação exclusiva. Revista Saúde Publica, 53:10.

Rydlewski, A. A., Pizzo, J. S., Manin, L. P., Zappielo, C. D., Galuch, M. B., Santos, O. O., \& Visentainer, J. V. (2020). Métodos Analíticos Utilizados para a Determinação de Lipídios em Leite Humano: Uma Revisão. Revista Virtual de Quimica. 12, 1-18.

Salustiano, L., Diniz, A., Abdallah, V., \& Pinto, R. (2012). Fatores associados à duração do aleitamento materno em crianças menores de seis meses. Rev Bras Ginecol Obstet., 28-3.

Sampaio, R., Sousa, L., \& M. S. C. (2017). Intolerância a lactos e vs. alergia a proteína do leite de vaca: a importância dos sinais e sintomas. Nutrição Brasil $16(2), 111-116$

Santana, J., Brito, S., \& Santos, D. (2013). Amamentação: conhecimento e prática de gestantes. O Mundo da Saúde, São Paulo, $259-267$.

Santana, S. M. (2019). Orientação Profissional Quanto Ao Aleitamento Materno: O Olhar Das Puérperas Em Uma Maternidade De Alto Risco No Estado De Sergipe. Enferm. Foco, 134-139.

Santos, Z. D. (2018). Benefícios do aleitamento materno exclusivo para o lactente e para a nutriz até o sexto mês. Revista Científica Multidisciplinar Núcleo do Conhecimento Ano 03, 07(02), 84-109.

Silva, A., Monteiro, G., Tavares, A., \& Pedrosa, Z. (2019). A introdução alimentar precoce e o risco de alergias: Revisão da literatura. Revista Electrónica trimestral de enfermagem, 485-498.

Silva, A., Ribeiro, K., Melo, L., Bezerra, D., Queiroz, J., Lima, M., \& Dimenstein, R. (2017). Vitamina e no leite humano e sua relação com o requerimento nutricional do recém-nacido a termo. Revista Paulista Pediatrico. 35(2), 158-164.

Souza, M. T., Silva, M. D., \& Carvalho, R. (2010). Revisão integrativa: o que é e como 15 fazer. Einstein, 8(1), 102-106.

Soares, R., \& Machado, J. (2012). Imunidade conferida pelo leite materno. Científica Univiçosa - 3(1), Viçosa-MG, $205-210$.

Tessari, W., Soares, L., Soares, L., \& Abreu, I. (2019). Percepção de mães e pais adolescentes sobre o aleitamento materno. Enferm. Foco, 10 (2), 83-89. 